\title{
Ethylenediaminetetraacetic acid: A non antibiotic adjuvant enhancing Pseudomonas aeruginosa susceptibility
}

\author{
Chaudhary Manu* and Payasi Anurag \\ Department of Cell Culture and Molecular Biology, Venus Medicine Research Centre, Hill Top Industrial Estate, Bhatoli \\ Kalan, Baddi, H.P. - 173205, India. \\ Accepted 3 September, 2012
}

\begin{abstract}
Pseudomonas aeruginosa is an opportunistic bacterium which has been shown to have multi-drug resistance against fluoroquinolones, $\beta$-lactams, and aminoglycosides. In this investigation, we studied the effect of different concentrations of ethylenediaminetetraacetic acid (EDTA) on MexA-MexB-OprM efflux pump and subsequent changes in susceptibility and expression. Next, we examined, the expression of mexAB gene following treatment with half of minimum inhibitory concentration (MIC) of drugs. Our results revealed that $10 \mathrm{mM}$ EDTA significantly reduced MIC of all drugs; moreover, the higher reduction (8 fold) was observed with CSE1034. MexA and MexB expression was down regulated at 2.93 and 3.21 fold, respectively with $10 \mathrm{mM}$ EDTA. When the same concentration of EDTA was incorporated with drugs, the CSE1034 down regulates 5.64 and 5.94 fold expression of $m e x A$ and $m e x B$, respectively. Moreover, meropenem treated groups exhibited 2.63 and 3.12 fold down regulation in the expression of the genes. However, treatment with piperacillin plus tazobactam, amoxicillin plus clavulanate, cefoperazone plus sulbactam and imipenem plus cilastatin did not produce changes in the expression of MexAB-OprM. Hence, CSE1034 could be one of the best choices to treat infections caused by microorganisms that overexpressed MexAB-Opr-M as compared to other drugs. Furthermore, use of EDTA disodium at appropriated concentrations can be regarded as a safe strategy to fight against the menace provided by the efflux pumps.
\end{abstract}

Key words: Amoxicillin plus clavulanate, CSE1034, ethylenediaminetetraacetic acid (EDTA), efflux pump, meropenem, Pseudomonas aeruginosa, piperacillin plus tazobactam.

\section{INTRODUCTION}

Pseudomonas aeruginosa is a Gram-negative, opportunistic pathogen, mostly involved in nosocomial infections, particularly pneumonia, in chronically ill patients (Stover et al., 2000; Engel and Balachandran, 2009). $P$. aeruginosa is increasingly difficult to eradicate due to significant intrinsic resistance to a variety of antimicrobial agents including fluoroquinolones, $\beta$ lactams, and aminoglycosides (Dotsch et al., 2009).

${ }^{*}$ Corresponding author. E-mail: research@venusremedies.com. Tel: 91-1795-302005. Fax: 91-1795-302133.
Different molecular mechanisms are responsible for multidrug resistant phenotypes of $P$. aeruginosa. Antimicrobial resistance of $P$. aeruginosa may be due to $\beta$-lactamases chromosomal, plasmid or integron encoded or by alterations in outer membrane permeability (that is, the loss of OprD protein), the overexpression of active efflux systems and the synthesis of aminoglycoside modifying enzymes (phosphoryl transferases, acetyl transferases, and adenyl transferases), significantly contributes to resistance as well as structural alterations of topoisomerases II and IV leading to quinolone resistance. Simultaneous expression of these mechanisms may be responsible for the multidrug 
resistant phenotypes of $P$. aeruginosa (McGowan, 2006; Falagas and Bliziotis, 2007; Gooderham and Hancock, 2009; Ruiz-Martínez et al., 2011a, b).

Among the causes of antibiotic resistance in $P$. aeruginosa, efflux pumps play a key role. Several reports have pointed out that overexpression of efflux systems results in reduced susceptibility to a variety of antimicrobial agents (Sobel et al., 2005; Cao et al., 2004). Efflux pumps are transporters localized in the cytoplasmic membrane of bacteria. To date, four multidrug efflux pumps have been well characterized in $P$. aeruginosa: MexA-MexB-OprM, MexC-MexD-OprJ, MexE-MexFOprN, and MexX-MexY-OprM.

MexAB-OprM is the most relevant (Poole et al., 1996); the MexAB-OprM efflux system functions to expel quinolones, aminoglycosides, macrolides, tetracycline, chloramphenicol, novobiocin, and most $\beta$-lactams (Poole and Srikumar, 2000). In addition to antimicrobial agents, the MexAB-OprM pump provides resistance against nonantibiotic compounds (Papadopoulos et al., 2008).

Efflux pump inhibitors (EPIs), which are compounds that target the efflux activity and/or pump components, have been identified and recommended as novel agents to fight against drug efflux mechanisms of pathogens (Page's et al., 2005). Susceptibility of $P$. aeruginosa strains to antibiotics can be enhanced by inactivation of one or more efflux pumps. A number of efflux pump inhibitors have been studied including phenylalanine arginyl $\beta$-naphthylamide (PA $\beta N$ ), carbonyl cyanide mchlorophenylhydrazone (CCCP), chlorpromazine, and verapamil (Askoura et al., 2011; Lechner et al., 2008; Rodrigues et al., 2008). Studies have found that PABN is an effective EPI as it is capable of potentiating fluoroquinolone activity in resistant $P$. aeruginosa strains. However, all these inhibitors have not yet met the requirements for application in clinical practice due to toxicity, immunosuppression, stability, and solubility concerns (Lomovskaya et al., 2001). Therefore, there is an urgent need of an efflux pump inhibitor which is safe and can be used in humans.

In the present investigation, we explored the effect of different concentrations of ethylenediaminetetraacetic acid (EDTA) on MexA-MexB-OprM efflux pump and subsequent changes in susceptibility and expression. Next, we examined, the expression of mexA and mexB genes following treatment with half of minimum inhibitory concentration (MIC) of drugs including CSE1034, piperacillin + tazobactam and amoxicillin plus clavulanate, cefoperazone plus sulbactam, imipenem plus cilastatin and meropenem.

\section{MATERIALS AND METHODS}

\section{Antimicrobial agents}

A novel antibiotic adjuvant entity (AAE) comprising ceftriaxone sodium, EDTA disodium and sulbactam sodium herein after referred to as CSE1034 (Venus Remedies Limited, Chandigarh, India), piperacillin plus tazobactam (Zosyn; Wyeth Pharmaceuticals, India, Mumbai) and amoxicillin plus clavulanate (Augmentin, Glaxo Smith Kline, Pharmaceuticals Ltd., Mumbai, India), cefoperazone plus sulbactam (Magnex, Pfizer, Mumbai, India), imipenem plus cilastatin (Tinaxim, New Medicon Pharma lab, Chennai, India), and meropenem (Meronem, Astrazeneca Pharma India Ltd., Banglore, India) were used in the study. All the drugs except CSE1034 were procured from the market. EDTA disodium was an essential non antibiotic adjuvant to CSE1034. EDTA disodium tested separately was procured from Akzo Nobel Functional Chemicals B.V. Stationsstraat 77, $3811 \mathrm{MH}$, P.O. Box 247, 3800 AE Amerfoort, Netherlands. All the drugs were reconstituted with water for injection according to instructions of the manufacturer. Working solutions were prepared using Mueller-Hinton $(\mathrm{MH}$, Himedia, Bombay, India) broth at a concentration from 0 to $8192 \mu \mathrm{g} / \mathrm{ml}$ and from these working solutions, serial two fold dilutions were made using Cation-Adjusted Mueller-Hinton (CAMH, Himedia, Bombay, India) broth in wells of 96-well plate.

\section{Studied organisms}

MexA-MexB-OprM overproducing K1455 (nalB mutant) and the MexA-MexB-OprM-deficient K1523 have been used in the study. The strains were procured from Dr. Keith Poole, Canada. LuriaBertani (LB) broth was used as the growth medium and bacteria were cultivated at $37^{\circ} \mathrm{C}$.

Susceptibility study of EDTA in different concentrations with drugs

Susceptibility testing to EDTA alone and each drug with concentrations of EDTA disodium ranging from 1 to $15 \mathrm{mM}$ was conducted on planktonic cultures of $P$. aeruginosa strains K1523 and K1455, according to the Clinical and Laboratory Standards Institute's Guidelines (2009). Working solutions were prepared in LB broth at a concentration of 0 to $8192 \mu \mathrm{g} / \mathrm{ml}$, and from these working solutions, serial two fold dilutions were made in CAMHB in wells of 96 -well plate.

\section{Total RNA extraction}

Total RNA was isolated with TRIzol (Invitrogen, Banglore, India). The amount of extracted RNA was quantified by measuring the absorbance at $260 \mathrm{~nm}$. The purity of the RNA was checked by measuring the ratio of the absorbance at 260 and $280 \mathrm{~nm}$, where a ratio ranging from 1.8 to 2.0 was taken to be pure. The absence of degradation of the RNA was confirmed by RNA electrophoresis on a $1.5 \%$ agarose gel containing ethidium bromide $(\mathrm{EtBr})$.

\section{Reverse transcription (RT) and polymerase chain reaction (PCR) analysis}

First-strand cDNA was generated from $2 \mu \mathrm{g}$ of each treatment using the reverse transcriptase kit (Invitrogen, Banglore, India) according to the manufacturer's protocol. PCR was performed in a total volume of $20 \mu \mathrm{l}$ containing $5 \mu \mathrm{l}$ of cDNA and per-designed, genespecific primers. Primers obtained from Sigma Aldrich Chemicals Pvt. Ltd., Bangalore, India. Primers used for mexA-5'GCCATGCGTGTACTGGTTCC-3' and mexA-5'TGACGTCGCTGCCTTCCTT; for mexB-5'-GTGTTCGGCTCGCA 
GTACTC-3' and mexB-5'-AACCGTCGGGATTGACCTTG-3'; for GAPDH-5'-ACTTACGAGCAGATCAAAGC-3' and GAPDH-5'AGTTTCACGAAGTTGTCGTT-3'.

The thermal cycling condition consisted of an initial PCR activation step at $95^{\circ} \mathrm{C}$ for $15 \mathrm{~min}$ followed by 35 cycles of denaturation $\left(94^{\circ} \mathrm{C}, 30 \mathrm{~s}\right)$, annealing $\left(55\right.$ to $60^{\circ} \mathrm{C}$ for $60 \mathrm{~s}$, depending on optimized conditions for the primers used) and extension $\left(72^{\circ} \mathrm{C}\right.$ for $\left.60 \mathrm{~s}\right)$. A $500-n g$ aliquot of total RNA was reverse-transcribed under similar conditions followed by PCR amplification of glyceraldehyde-3-phosphate dehydrogenase (GAPDH), cDNA for 35 cycles. PCR products were analyzed by $2 \%$ agarose gel electrophoresis. The housekeeping gene GAPDH was used as a control template for normalizing relative changes of mexAB mRNA in RT-PCR.

\section{Effect of EDTA on mexA and mexB expression}

To assess the effect of EDTA disodium on gene expression, the $P$. aeruginosa MexA-MexB-OprM-overexpressing strain was treated for $24 \mathrm{~h}$ with different concentration of EDTA ranging from 1 to 15 $\mathrm{mM}$ and total RNA was isolated and then first strand cDNA was synthesized for further study. The same strain without EDTA treatment serves as a control.

\section{Effect of different drugs on mexA and mexB expression}

To assess the effect of drugs on expression of efflux pump, the $P$. aeruginosa MexA-MexB-OprM positive culture was treated with different drugs at half of MIC for $24 \mathrm{~h}$ (Table 4). Following treatment, total RNA was isolated with TRIzol (Invitrogen). First strand cDNA was then synthesized from $2 \mu \mathrm{g}$ total RNA using reverse transcriptase (Invitrogen). The same strain without drug treatment serves as a control.

\section{Reproducibility of the results}

All results were carried out in triplicate and representative data are presented.

\section{Statistical analysis}

Data were analyzed using Graph Pad prism 5.01 and expressed as mean \pm standard deviation (SD). The continuous variables were tested with one-way analysis of variance (ANOVA) and Dunnet test. Values less than 0.05 were considered statistically significant.

\section{RESULTS}

\section{Susceptibility study of EDTA in different concentrations with drugs}

Results as shown in Table 1 , revealed that the MIC of EDTA disodium alone against strains $\mathrm{K} 1455$ and $\mathrm{K} 1523$ was 4096 and $2048 \mu \mathrm{g} / \mathrm{ml}$, respectively. The addition of different concentrations of EDTA resulted in a decrease in MIC values with maximum reduction at $10 \mathrm{mM}$ for all drugs. Moreover, MIC of CSE1034 reduced 8 fold $(\mu \mathrm{g} / \mathrm{ml})$ in MexA-MexB-OprM over expressing strain. On the other hand, 4 fold decreases in MIC values was noted with piperacillin plus tazobactam, imipenem plus cilastatin and meropenem, whereas 2 fold decrease was observed with amoxicillin plus clavulanate and cefoperazone plus sulbactam. However, 4 fold decreases in MIC with MexAB-OprM deficient strain was noted for all drugs (Table 2).

\section{Effect of EDTA on expression of mexA and mexB}

Our results revealed that mexA and mexB had higher expression in control group, which was not treated with EDTA. The down-regulation of the expression of the mexA and mexB following treatment with EDTA occurred in dose dependent manner with 2.93 and 3.21 fold reduction, respectively, at $10 \mathrm{mM}$. Further increasing the concentration of EDTA failed to produce significant changes in the expression (Table 3).

\section{Effect of different drugs on expression of mexA and mexB}

Our results revealed that the expression of mexA and mexB in CSE1034 treated group was down regulated approximately at 5.64 and 5.94 fold, respectively as compared to the control. Moreover, meropenem treated groups exhibited 2.63 and 3.12 fold down regulation in the expression of mexA and mexB genes, respectively. While other comparative groups including imipenem plus cilastatin, piperacillin plus tazobactam, amoxicillin plus clavulanate, and cefoperazone plus sulbactam did not produce any significant changes of the expression (Figures 1 and 2).

\section{DISCUSSION}

Both specific and unspecific efflux mechanisms play important role in intrinsic and/or acquired resistance to antimicrobials. The $P$. aeruginosa genome contains at least 10 distinct tripartite (3-protein) efflux pump system gene operons (Stover et al., 2000).

Efflux pump inhibition may be due to disturbance of the regulatory pathway essential for expression of the efflux pump, alteration of the chemical structure of the antibiotic preventing its attachment to a specific substrate, hindrance of assembly of efflux pump components, inhibition of antibiotic by completive or non-completive binding, closing of the pores responsible for efflux of antimicrobial agents or disturbance of energy essential for pump activity (Poole and Lomovskaya, 2006; Pagés and Amaral, 2009).

As pointed out in this study, EDTA reduced MICs at concentrations up to $10 \mathrm{mM}$, although higher concentrations did not produce higher inhibitions in overexpressing isolates. On the contrary, a certain reduction 
Table 1. Effect of EDTA on susceptibility of $P$. aeruginosa strains with different expression of the MexAB-OprM efflux pump.

\begin{tabular}{|c|c|c|c|}
\hline \multirow{2}{*}{ Antibiotic } & \multirow{2}{*}{ EDTA (mM) } & \multicolumn{2}{|c|}{ MIC $(\mu \mathrm{g} / \mathrm{ml})$} \\
\hline & & MexAB-OprM-overexpressed & MexAB-OprM-deficient \\
\hline \multirow{5}{*}{$\begin{array}{l}\text { Ceftriaxone plus sulbactam } \\
\text { (CSE1034) }\end{array}$} & None & 8 & 0.0624 \\
\hline & 1 & 8 & 0.0624 \\
\hline & 5 & 4 & 0.031 \\
\hline & 10 & 1 & 0.0156 \\
\hline & 15 & 1 & 0.0156 \\
\hline \multirow{5}{*}{ Piperacilline plus tazobactam } & None & 32 & 2 \\
\hline & 1 & 32 & 2 \\
\hline & 5 & 16 & 1 \\
\hline & 10 & 8 & 0.5 \\
\hline & 15 & 8 & 0.25 \\
\hline \multirow{5}{*}{ Amoxicillin plus clavulanate } & None & $>128$ & 2 \\
\hline & 1 & $>128$ & 2 \\
\hline & 5 & 128 & 1 \\
\hline & 10 & 64 & 0.5 \\
\hline & 15 & 64 & 0.5 \\
\hline \multirow{5}{*}{ Cefoperazone plus sulbactam } & None & 64 & 4 \\
\hline & 1 & 64 & 4 \\
\hline & 5 & 64 & 2 \\
\hline & 10 & 32 & 1 \\
\hline & 15 & 32 & 1 \\
\hline \multirow{5}{*}{ Imipenem plus cilastatin } & None & 64 & 1 \\
\hline & 1 & 64 & 1 \\
\hline & 5 & 32 & 0.5 \\
\hline & 10 & 16 & 0.25 \\
\hline & 15 & 16 & 0.125 \\
\hline \multirow{5}{*}{ Meropenem } & None & 64 & 1 \\
\hline & 1 & 64 & 1 \\
\hline & 5 & 32 & 0.5 \\
\hline & 10 & 16 & 0.25 \\
\hline & 15 & 16 & 0.125 \\
\hline EDTA & & 4096 & 2048 \\
\hline
\end{tabular}

Table 2. Fold reduction of MIC at $10 \mathrm{mM}$ of EDTA in $P$. aeruginosa strains.

Name of antibiotic

Ceftriaxone plus sulbactam (CSE1034)

Piperacilline plus tazobactam

Amoxicillin plus clavulanate

Cefoperazone plus sulbactam

Imipenem plus cilastatin

Meropenem

Fold reduction in MIC ( $\mu \mathrm{g} / \mathrm{ml})$

MexAB-OprM-overexpressed

MexAB-OprM-deficient

$\begin{array}{ll}8 & 4 \\ 4 & 4 \\ 2 & 4 \\ 2 & 4 \\ 4 & 4 \\ 4 & 4\end{array}$


Table 3. Effect of EDTA on expression of mexA and mexB genes in $P$. aeruginosa.

\begin{tabular}{ccc}
\hline Concentrations of EDTA $(\mathbf{m M})$ & mexA & mexB \\
\hline 0 & $91.11 \pm 8.7$ & $90.04 \pm 9.1$ \\
1 & $87.65 \pm 7.5$ & $86.32 \pm 7.1$ \\
5 & $54.31 \pm 5.1$ & $46.51 \pm 5.1$ \\
10 & $31.06 \pm 2.8$ & $28.12 \pm 3.0$ \\
15 & $26.21 \pm 2.9$ & $22.41 \pm 2.1$ \\
\hline
\end{tabular}

Expression was determined by semi-quantitative RT-PCR, normalized to GAPDH. Results are presented in mean \pm SD. $0=$ without EDTA.

Table 4. Concentration of drugs used in the expression study.

\begin{tabular}{lcc}
\hline \multirow{2}{*}{ Name of antibiotic } & \multicolumn{2}{c}{ Concentration $(\boldsymbol{\mu g} / \mathbf{m I})$} \\
\cline { 2 - 3 } & MIC & Half of MIC \\
\hline Ceftriaxone plus sulbactam (CSE1034) & 1 & 0.5 \\
Piperacilline plus tazobactam & 8 & 4 \\
Amoxicillin plus clavulanate & 64 & 32 \\
Cefoparazone plus sulbactam & 32 & 16 \\
Imipenem plus cilastatin & 16 & 8 \\
Meropenem & 16 & 8 \\
\hline
\end{tabular}

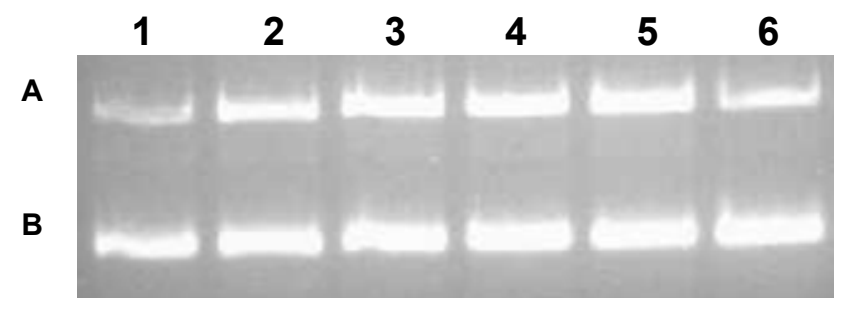

Figure 1. Effect of drugs on expression of mexA. A=Treatment groups where 1=CSE1034; 2=amoxicillin+clavulanate; $\quad 3=$ cefoperazone+sulbactam; 4=imipenem+cilastatin; $\quad 5=$ piperacillin+tazobactam; $6=$ meropenem; and $\mathrm{B}=$ Control group.

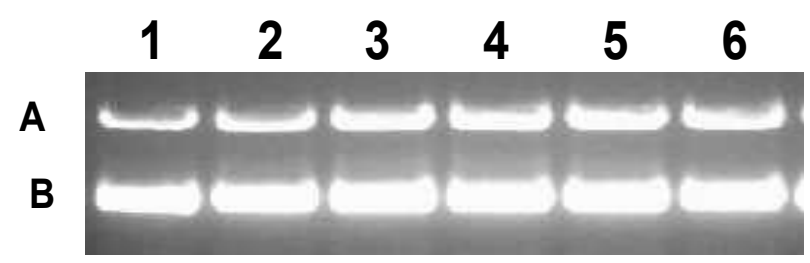

Figure 2. Effect of drugs on expression of mexB. $A=$ Treatment groups where $1=$ CSE1034; $2=$ meropenem, $3=$ amoxicillin+clavulanate;

4=Cefoperazone+Sulbactam;5=imipenem+cilastatin; 6=piperacillin+tazobactam; and B=Control group.

was observed in under-expressing strain for piperacillin plus tazobactam, meropenem and imipenem plus cilastatin. Efflux pump inhibitors not only reduce the MIC, but also reduce the invasiveness of $P$. aeruginosa, when used in combination with an antibiotic (Hirataka et al., 2009). Efflux pump inhibition by EDTA begins at a concentration of $5 \mathrm{mM}$ and continues up to $10 \mathrm{mM}$, confirming efflux pump inhibitory property. In CSE1034 due to synergistic action of trio (ceftriaxone sodium plus EDTA disodium plus sulbactam sodium), efflux pump inhibition is achieved at much lower concentration of EDTA. This concentration is achievable in-vivo and hence the efficacy of CSE1034 in efflux pump inhibition should be tested in clinical. EDTA disodium intravenous safety has been established by Dwivedi et al. (data not published) at $170 \mathrm{mg} / \mathrm{kg}$ body weight corresponding to $45.9 \mathrm{mM}$ concentration of EDTA.

The mexA and mexB exhibited 2.93 and 3.21 fold down regulation in the gene expression with $10 \mathrm{mM}$ EDTA, respectively. The down-regulation of $\operatorname{mex} A$ and $\operatorname{mex} B$ genes following treatment with EDTA disodium is probably due to chelation of divalent ions (Vaara, 1992), thereby disturbing the electrostatic interactions in the outer membrane of the bacterial cell and may be responsible for blocking of pores associated with efflux activity (Pagés and Amaral, 2009; Poole and Lomovskaya, 2006). Inhibition of the efflux pumps is promising in order to increase the intracellular drug concentration, restore the drug activity against the resistant strains, and minimize further development of resistant strains. Moreover, when drugs at half of MIC were studied, only CSE103 and meropenem exhibited 
the down regulation of mexA and mexB genes. However, the maximum expression reduction, 5.64 and 5.94 fold of mexA and mexB, respectively, was noted with CSE1034 suggesting that increased antibiotic accumulation within the bacteria was associated with reduced expression of mexA and mexB genes.

According to Schneider and Gourse (2004), the adenosine triphosphate (ATP) concentration in Gramnegative bacteria directly controls the rate of rRNA transcription initiation, thereby affecting protein synthesis and therefore growth rate. It is thus likely that, addition of EDTA to antibiotics as a non-antibiotic adjuvant helps in down regulating protein expression responsible for mexAMexB-OmpM, which in turn is responsible for efflux overexpression in $P$. aeruginosa (Chaudhar et al., unpublished).

In conclusion, results obtained in the present study indicate that $10 \mathrm{mM}$ EDTA disodium enhance the susceptibility of $P$. aeruginosa towards antibiotics by down regulating the expression of efflux pump. Furthermore, CSE1034 can be one of the best choices to treat infections caused by microorganisms that over expressing MexAB-Opr-M as compared to other drugs.

\section{ACKNOWLEDGEMENTS}

The authors are thankful to their sponsor, Venus Pharma $\mathrm{GmbH}$, AM Bahnhof 1-3, D-59368, Werne, Germany, for providing assistance to carry out this research. The authors are also thankful to Dr. Keith Poole for providing $P$. aeruginosa strains.

\section{REFERENCES}

Askoura M, Mottawea W, Turki A, Ibrahim T (2011). Efflux pump inhibitors (EPIs) as new antimicrobial agents against Pseudomonas aeruginosa. Libyan J. Med. 6:5870-5878.

Cao L, Srikumar R, Poole K (2004). MexAB-OprM hyperexpression in NalC-type multidrug-resistant Pseudomonas aeruginosa: identification and characterization of the nalC gene encoding a repressor of PA3720- PA3719. Mol. Microbiol. 53:1423-1436.

Clinical and Laboratory Standards Institute (CLSI) (2009). Performance standards for antimicrobial susceptibility testing, $19^{\text {th }}$ informational supplement, CLSI document M100-S19, Wayne, PA: CLSI, 29.

Dotsch A, Becker T, Pommerenke C, Magnowska Z, Jansch L, Haussler S (2009). Genomewide identification of genetic determinants of antimicrobial drug resistance in Pseudomonas aeruginosa. Antimicrob. Agents Chemother. 53:2522-2531.

Engel J, Balachandran P (2009). Role of Pseudomonas aeruginosa type III effectors in disease. Curr. Opin. Microbiol. 12:61-66.

Falagas ME, Bliziotis IA (2007). Pandrug-resistant gram negative bacteria: the dawn of the post-antibiotic era? Int. J. Antimicrob. Agents 29:630-636.
Gooderham WJ, Hancock REW (2009). Regulation of virulence and antibiotic resistance by two-component regulatory systems in Pseudomonas aeruginosa. FEMS Microbiol. Rev. 33:279-294.

Lechner D, Gibbons S, Bucar F (2008). Plant phenolic compounds as ethidium bromide efflux inhibitors in Mycobacterium smegmatis. J. Antiub. Chemother. 62:345-348.

Lomovskaya O, Warren MS, Lee A, Galazzo J, Fronko R, Lee M, Blais J, Cho D, Chamberland S, Renau T, Leger, Hecker S, Watkins W, Hoshino K, Ishida H, Lee VJ (2001). Identification and characterization of inhibitors of multidrug resistance efflux pumps in Pseudomonas aeruginosa: novel agents for combination therapy. Antimicrob. Agents Chemother. 45:105-116.

Pagés JM, Amaral L (2009). Mechanisms of drug efflux and strategies to combat them: challenging the efflux pump of gram-negative bacteria. Biochim. Biophys. Acta 1794:826-833.

Pagés JM, Masi M, Barbe J (2005). Inhibitors of efflux pumps in Gramnegative bacteria. Trends Mol. Med. 11:382-389.

Papadopoulos CJ, Christine FC, Barbara JC, Riley TV (2008). Role of the MexAB-OprM efflux pump of Pseudomonas aeruginosa in tolerance to tea tree (Melaleuca alternifolia) oil and its monoterpene components terpinen-4-ol, 1,8-cineole, and a-terpineol. Appl. Environ. Microbiol. 74:1932-1935.

Poole K, Lomovskaya O (2006). Can efflux inhibitors really counter resistance? Infect. Dis. 3:145-152.

Poole K, Srikumar R (2000). Multidrug efflux in Pseudomonas aeruginosa: components, mechanisms and clinical significance. Curr. Top. Med. Chem. 1:59-71.

Poole K, Tetro K, Zhao Q, Neshat S, Heinrichs DE, Bianco N (1996). Expression of the multidrug resistance operon mexA-mexB-oprM in Pseudomonas aeruginosa: mexR encodes a regulator of operon expression. Antimicrob. Agents Chemother. 40:2021-2028

Rodrigues L, Wagner D, Viveiros M, Sampaio D, Couto I, Vavra M, Kern WV, Amaral L (2008). Thioridazine and chlorpromazine inhibition of ethidium bromide efflux in Mycobacterium avium and Mycobacterium smegmatis. J. Antipb. Chemother. 61:1076-1082.

Ruiz-Martínez L, López-Jiménez L, d'Ostuni V, Fusté E, Vinuesa T, Viñas M. (2011a). A mechanism of carbapenem resistance due to a new insertion element (ISPa133) in Pseudomonas aeruginosa. Int Microbiol. 14:51-58.

Ruiz-Martínez L, López-Jiménez L, Fusté E, Vinuesa T, Martínez JP, Viñas M. (2011b). Class 1 integrons in environmental and clinical isolates of Pseudomonas aeruginosa. Int. J. Antimicrob. Agents 38:398-402

Schneider DA, Gourse RL (2004). Relationship between growth rate and ATP concentration in Escherichia coli. J. Biol. Chem. 279:82628268.

Sobel ML, Hocquet D, Cao L, Plesiat P, Poole K (2005). Mutations in PA3574 (nalD) lead to increased MexAB-OprM expression and multidrug resistance in laboratory and clinical isolates of Pseudomonas aeruginosa. Antimicrob. Agents Chemother. 49:17821786.

Stover CK, Pham XQ, Erwin AL, Mizoguchi SD, Warrener P, Hickey MJ, Brinkman FS, Hufnagle WO, Kowalik DJ, Lagrou M, Garber RL, Goltry L, Tolentino E, Westbrock-Wadman S, Yuan Y, Brody LL, Coulter SN, Folger KR, Kas A, Larbig K, Lim R, Smith K, Spencer D, Wong GK, Wu Z, Paulsen IT, Reizer J, Saier MH, Hancock RE, Lory $\mathrm{S}$, Olson MV (2000). Complete genome sequence of Pseudomonas aeruginosa PA01, an opportunistic pathogen (comment). Nat. 406:959-964.

Vaara M (1992). Agents that increase the permeability of the outer membrane. Microbiol. Rev. 56:395-4111. 